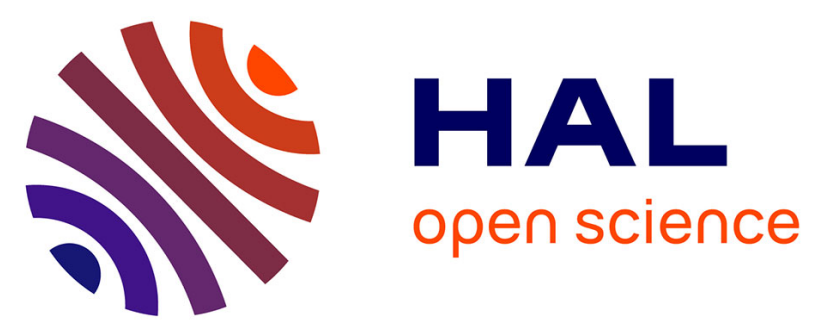

\title{
Modeling preasymptotic transport in flows with significant inertial and trapping effects - The importance of velocity correlations and a spatial Markov model
} Diogo Bolster, Yves Méheust, Tanguy Le Borgne, Jeremy Bouquain, Philippe Davy

\section{To cite this version:}

Diogo Bolster, Yves Méheust, Tanguy Le Borgne, Jeremy Bouquain, Philippe Davy. Modeling preasymptotic transport in flows with significant inertial and trapping effects - The importance of velocity correlations and a spatial Markov model. Advances in Water Resources, 2014, 70, pp.89-103. 10.1016/j.advwatres.2014.04.014 . insu-01004059

HAL Id: insu-01004059

https://hal-insu.archives-ouvertes.fr/insu-01004059

Submitted on 11 Jun 2014

HAL is a multi-disciplinary open access archive for the deposit and dissemination of scientific research documents, whether they are published or not. The documents may come from teaching and research institutions in France or abroad, or from public or private research centers.
L'archive ouverte pluridisciplinaire HAL, est destinée au dépôt et à la diffusion de documents scientifiques de niveau recherche, publiés ou non, émanant des établissements d'enseignement et de recherche français ou étrangers, des laboratoires publics ou privés. 


\title{
Modeling preasymptotic transport in flows with significant inertial and trapping effects - the importance of velocity correlations and a spatial Markov model
}

\author{
Diogo Bolster ${ }^{\mathrm{a}}$, Yves Méheust ${ }^{\mathrm{b}}$, Tanguy Le Borgne ${ }^{\mathrm{b}}$, Jérémy Bouquain $^{\mathrm{b}}$, \\ Phillipe Davy ${ }^{\mathrm{b}}$ \\ ${ }^{a}$ Environmental Fluid Dynamics Laboratories, Dept. of Civil and Environmental \\ Engineering and Earth Sciences, University of Notre Dame, IN, USA \\ ${ }^{b}$ Geosciences Rennes (UMR CNRS 6118), Université Rennes 1, Campus de Beaulieu, \\ 35042 Rennes Cedex, France
}

\begin{abstract}
We study solute transport in a periodic channel with a sinusoidal wavy boundary when inertial flow effects are sufficiently large to be important, but do not give rise to turbulence. This configuration and setup are known to result in large recirculation zones that can act as traps for solutes; these traps can significantly affect dispersion of the solute as it moves through the domain. Previous studies have considered the effect of inertia on asymptotic dispersion in such geometries. Here we develop an effective spatial Markov model that aims to describe transport all the way from preasymptotic to asymptotic times. In particular we demonstrate that correlation effects must be included in such an effective model when Péclet numbers are larger than $\mathrm{O}(100)$ in order to reliably predict observed breakthrough curves and the temporal evolution of second centered moments. For smaller Péclet numbers correlation effects, while present, are weak and do not appear to play a significant role. For many systems of practical interest, if Reynolds numbers are large, it may be typical that Péclet numbers are large also given that Schmidt numbers for typical fluids and solutes can vary between 1500. This suggests that when Reynolds numbers are large, any effective theories of transport should incorporate correlation as part of the upscaling procedure, which many conventional approaches currently do not do. We define a novel parameter to quantify the importance of this correlation. Next, using the the-
\end{abstract}


ory of CTRWs we explain a to date unexplained phenomenon of why dispersion coefficients for a fixed Péclet number increase with increasing Reynolds number, but saturate above a certain value. Finally we also demonstrate that effective preasymptotic models that do not adequately account for velocity correlations will also not predict asymptotic dispersion coefficients correctly.

Keywords: Inertial Flow, Upscaling Transport, Spatial Markov

\section{Introduction}

GI Taylor [44], in a seminal work, demonstrated that at asymptotic times, for laminar flow conditions transport in a cylindrical tube can be effectively described by a one dimensional transport equation with an enhanced longitudinal effective dispersion that accounts for the interplay between transverse molecular diffusion and longitudinal advection by the shear flow. Aris then formalized this concept by relating this dispersion coefficient to the second centered spatial moment of a plume [3]. For all parallel shear flow geometries, the scaling of the dispersion coefficient as a function of the average fluid velocity, typical geometrical dimension, and molecular diffusion coefficient is the same. See for example [16] for a detailed account of Taylor's, Aris', and subsequent early developments on this matter. Since then there have been countless studies, in numerous fields including hydrology, using effective dispersion coefficients, thus demonstrating the strength and utility of this simple concept. While Taylor's concept was limited to purely shear flows, the idea was extended to more complex domains and flows by a variety of methods, including the method of moments [17], the method of volume averaging [41] and homogenization [31]. Howard Brenner, in

honor of Taylor's contribution, coined the term 'Taylor dispersion' to describe this effective dispersion phenomenon.

Taylor dispersion is only valid at asymptotic times, which in other words means after the solute in the system has had sufficient time to sample the full statistics of the velocity field. This time scale is determined by how quickly diffusion can act and is typically quantified as $\tau_{D}=l_{c}^{2} / D$, where $l_{c}$ is a charac- 
teristic length scale of the system being considered. If this time is much shorter than the times of interest, then it may be appropriate to use asymptotic Taylor dispersion estimates. However, if the time scales of interest are shorter than or on the order of $\tau_{D}$, then preasymptotic effects should be accounted for. At preasymptotic times the evolution of the spatial moments of a plume generally do not scale in a Fickian manner and so models capable of capturing these anomalous scalings should be used. For the specific case of shear flows considered by Taylor and Aris a variety of papers studying preasymptotic effects have been published [29, 9, 36, 16]. Pre asymptotic effects may be particularly important for modeling mixing, since transverse concentration gradients are significant in this regime $[36,37,12]$. The goal of the work presented here is to develop and test a model for transport at preasymptotic times.

For more complex geometries and flows traditional Taylor-Aris type modeling approaches may not be appropriate, but a rich family of alternatives exists. In this particular work we will focus on the Spatial Markov model originally introduced and studied in $[13,14,33,1]$ to model effective transport in highly heterogeneous porous media flows. This model has also gone by the name correlated CTRW and has been applied successfully to model effective preasymptotic transport in a channel with periodically varying aperture and in a two-dimensional porous medium at the pore scale $[38,1]$. Both of these flows were considered using the classical porous medium assumption of Stokes flow at low Reynolds numbers $(R e \ll 1)$.

While for many practical applications the low Reynolds assumption is perfectly reasonable, there are situations where inertial effects may play an important role, e.g. flow through fractures [19], or flows where fluid viscosity is small such as for Carbon sequestration where the viscosity of supercritical $\mathrm{CO}_{2}$ can be two orders of magnitude smaller than that of water [5, 45]. Inertial effects change the structure of the flow, which can have profound implications when upscaling the flow equation. In particular, as inertial effects increase, the presence of recirculation zones also increases. While such recirculation zones can and do occur for flows at low (and even vanishing) Reynolds number [40, 35, 10] 
they become more pronounced as inertial effects become increasingly important; their structure can also change significantly with increasing inertia, leading for example to strong asymmetries with respect to the flow direction, regardless of existing symmetries in the geometry. These recirculation zones are low velocity regions that are known to have a significant impact on conservative $[30,20,8]$ and reactive transport $[27,48]$. They are often conceptualized as regions where solutes are trapped in an immobile state. For example, Aris [4] studied asymptotic dispersion coefficients in flow through a tube with immobile pockets.

In this work, we study preasymptotic transport in a system where inertial effects in the flow are important. We focus on a simple idealized pore geometry, namely a channel with a periodically varying aperture, which is formally defined in the following section. While this is a very simple system it has been shown in a variety of studies to provide valuable physical insight into what controls flow and transport in a porous medium. One of the benefits is that the system is sufficiently simple so as to limit the number of relevant dimensionless numbers, a feature exploited in a variety of previous studies. Limitations of this geometry are that it is periodic, which true porous media will likely not be, it is vertically bounded meaning that transverse dispersion effects cannot be explored and it is two-dimensional, where real systems would be three dimensional. Dykaar \& Kitanidis [28] chose this geometry to upscale reactive transport in a porous medium. Cardenas et al. [18] studied a similar geometry in a variety of contexts to assess the impact of inertia on flow from the perspective of upscaling flow and the breakdown of Darcy's law [22]. Bolster et al. [10] used this geometry to demonstrate that an increase in boundary fluctuations does not automatically induce greater dispersion under creeping flow conditions. Le Borgne et al [38] used this geometry to study preasymptotic transport in low Reynolds number flows and the authors showed that the features that emerged from this simple geometry were both qualitatively and quantitatively similar to features obtained from a more complex, heterogeneous and realistic pore structure. Bouquain et al [15] studied asymptotic dispersion in this geometry when inertial effects become important. They investigated the dependence of the asymptotic effective 
dispersion coefficient on Reynolds and Péclet, and observed that it saturates at high Reynolds numbers at a Péclet-dependent value. A scaling law was found for the asymptotic dispersion coefficient as a function of Reynolds and Péclet, with a power law dependence on the Péclet that deviates from the classic $P e^{2}$ of Taylor and Aris. A recent work by Richmond et al [42] looks at asymptotic dispersion in a similar geometry, including at higher Reynolds number unsteady, but not fully turbulent, flow. At these larger Reynolds number temporal velocity fluctuations result in increased mass transfer, diminishing tailing and thus decreasing longitudinal dispersion effects. It is some of these results that motivate the current study, where we look more closely into the role of recirculation zones. Wood [46], using the theory of volume averaging, studied dispersion in flows through porous media where inertial effects play a role and showed how different scalings with Péclet number for the asymptotic dispersion coefficient emerge depending on the specific value of the Reynolds number and whether inertial effects are important or not. Experiments exploring asymptotic longitudinal dispersion in porous media where inertial effects are significant have also been conducted [23], showing scalings similar to those in [46].

The paper is structured as follows. Section 2 defines the model system, the numerical methods used to study flow and transport through it, the development of the proposed effective spatial Markov model and the metrics and observables used to test the model. Section 3 presents results of the simulation and comparisons of observed results to model predictions. Section 4 provides a discussion on predictions of asymptotic dispersion coefficients using CTRW theory which does not incorporporate correlations and demonstrates how preasymptotic models that do not adequately account for correlation effects will not accurately predict asymptotic dispersion coefficients for sufficiently large Péclet numbers. It also discusses the importance of the probability of successive trapping of particles in immobile/recirculation zones. Section 5 provides conclusions of this work. 


\section{Model System}

\subsection{Geometry}

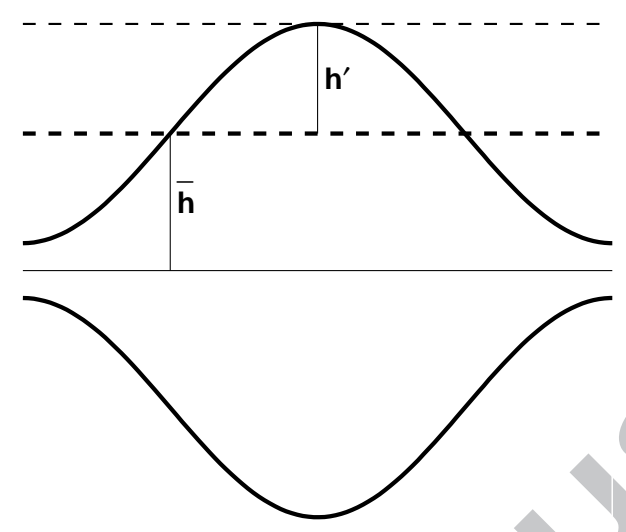

Figure 1: Schematic of the unit cell 'pore' for flow and transport modeling in this work.

The geometry we consider in this study is a two-dimensional channel with a sinusoidal boundary, depicted in figure 1 ; the boundaries of the system are described by

$$
h(x)=\bar{h}-h^{\prime} \cos \left(\frac{2 \pi x}{L}\right),
$$

where $x$ is the horizontal coordinate, $h$ is the half-aperture, $\bar{h}$ is the mean half-aperture, $h^{\prime}$ is the amplitude fluctuation of the half-aperture and $L$ is the length of a single cell. This geometry is fully characterized by two dimensionless numbers

$$
\epsilon=\frac{2 \bar{h}}{L} \quad \text { and } \quad a=\frac{h^{\prime}}{2 \bar{h}}
$$

where $\epsilon$ describes the aspect ratio of the cell and $a$ the relative amplitude of the aperture fluctuations. $a$ is bounded between $0<a<1 / 2$ as for values equal to or greater than $1 / 2$ the channel is closed and no flow occurs. For $a=0$ one recovers Poiseuille flow, i.e. parallel flow between two plates. Bouquain et al 
[15] demonstrated that for sufficient $a$ and $\epsilon$ recirculation zones will arise even if they were not present at $R e=0$ and grow in area as inertial effects become more important. In order to maintain a small number of dimensionless parameters in this current study we will restrict ourselves to a system with specific values of $a=0.4$ and $\epsilon=0.47$. These values are chosen because they coincide with one set of values that received focus in [15]. However, as outlined in [15], the features that we will focus on in this study such as emergence of recirculation zones as inertial effects increase or an asymptote in the effective dispersion coefficient as inertial effects increase are not restricted to these values and occur for similar reasons at other values of $\epsilon$ and $a$.

We consider flow in this system from left to right. Given a mean fluid velocity $\bar{u}$ we define the additional dimensionless Reynolds number as

$$
R e=\frac{2 \bar{h} \bar{u}}{\nu}
$$

where $\nu$ is the kinematic viscosity of the fluid. The Reynolds number characterizes the relative importance of inertial to viscous effects. In this study we always maintain $\bar{h}$ and $\bar{u}$ constant and so modify $\nu$ to change the Reynolds numbers so that systems with similar average velocities can be easily compared. As noted, in this study we are interested in flows where inertial effects are important and so we focus on flows for which $R e \geq 1$. However, in this current study we are not interested in the role of turbulence, which emerges at very large Reynolds numbers. We restrict our study to non-turbulent inertial effects with Reynolds number smaller than 100. While in [15] we studied a broad range of $1 \leq R e \leq 100$, here we focus primarily on cases where $R e=\{1,30,100\}$ as these effectively serve to highlight our central messages. Streamlines for these three cases are shown in figure 2. For $R e=1$ no recirculation is present and streamlines follow the wall boundary structure. For $R e=30$ a large asymmetric recirculation zone has already developed. For $R e=100$ a very large recirculation zone that occupies most of the domain, other than a narrow flow channel down the center between throats, exists. 
Since we are interested in describing the effective transport of a solute, a final dimensionless number, the Péclet number, arises such that

$$
P e=\frac{2 \bar{h} \bar{u}}{D}
$$

where $D$ is the diffusion coefficient of the solute considered. The Péclet number is a measure of the ratio of advective to diffusive effects in transport. In this it is similar to the Reynolds number, which is an estimate of the same ratio for the flow. The ratio of the Péclet number to Reynolds number is known as the Schmidt number $S c=P e / R e=\nu / D$; it is an intrinsic property of the fluid and solute and for most solutes of practical interest it is greater than 1 , meaning that when advective effects are important for flow they are also important for transport.

\subsection{Flow simulations}
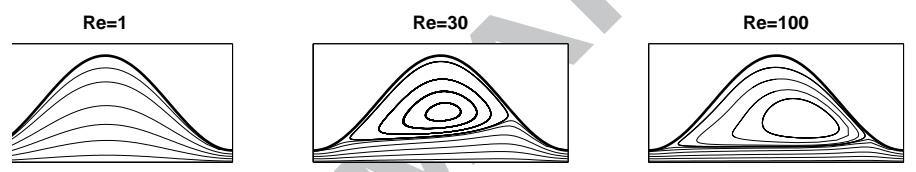

Figure 2: Streamlines for the selected geometry at $R e=1,30$ and 100. Note the larger area occupied by the recirculation zones as the Reynolds number gets bigger and bigger.

The steady state flow field through the geometry described above was modeled numerically by direct numerical simulation. The flow was treated as incompressible, resulting in the following conservation of mass and momentum equations (Navier-Stokes)

$$
\boldsymbol{\nabla} \cdot \mathbf{u}=0 \quad \rho(\mathbf{u} \cdot \nabla \mathbf{u})=\rho \mathbf{g}-\nabla p+\mu \nabla^{2} \mathbf{u}
$$

These equations are solved using a finite element method with the commercially available software package COMSOL MULTIPHYSICS. The in and outflow boundaries are treated as periodic, a mean flow is imposed at the inlet and a constant pressure at the outlet, which allows the solver to adjust the 
pressure so as to satisfy periodicity and the imposed flow rate. Further details are available in [15].

\subsection{Simulation of transport}

We consider conservative solute transport as governed by the advection diffusion equation

$$
\frac{\partial C}{\partial t}+\mathbf{u} \cdot \nabla C=D_{\mathrm{m}} \nabla^{2} C
$$

where $C$ is the concentration of the solute and $D_{\mathrm{m}}$ is the molecular diffusion coefficient. In all cases we consider a flux weighted pulse initial condition at the narrowest location in the throat of the channel. Due to the linearity of the problem at hand this initial condition provides sufficient information to predict transport for more complex initial configurations, although it should be noted that specific breakthrough curves and spatial moments of a plume can be sensitive to the specific initial condition [21, 47, 49]. We solve this system numerically using a Lagrangian particle based random walk method, where the solute plume is discretized into a finite number of $N$ particles. In our simulations particle numbers were increased until results converged. Generally this was achievable with $10^{4}$ particles and to minimize noise effects in most cases we used one million particles. For a given time step $\Delta t$ each particle $i$ is then moved according to the Langevin equation

$$
\begin{aligned}
& x_{i}(t+\Delta t)=x_{i}(t)+u_{i} \Delta t+\xi_{i} \sqrt{2 D \Delta t} \\
& y_{i}(t+\Delta t)=y_{i}(t)+v_{i} \Delta t+\eta_{i} \sqrt{2 D \Delta t}
\end{aligned} \quad i=1, \ldots, N,
$$

where $x_{i}$ and $y_{i}$ are the horizontal and vertical position of particle $i$ respectively and $\xi$ and $\eta$ are independently distributed Gaussian variables with zero mean and unit variance. We used a third order Runge-Kutta scheme to advance particle locations in time. The choice of time step is critical in producing accurate results (e.g. [32]). Typically we found convergence of results for time steps smaller than $\Delta t=10^{-2}$ and in most cases ran simulations with steps of $\Delta t=10^{-3}$. It should be noted that time in these simulations is chosen such 
that one unit of time corresponds to the amount of time it takes an advective particle travelling with the mean velocity $\bar{u}$ to traverse a distance $2 h$. The solid boundaries in the domain are treated as no flux boundaries and are modeled numerically as elastic reflection boundaries for particles, meaning that we calculate a particle's angle of incidence as it strikes a boundary and reflect it with the same angle of reflection. Particle velocities are interpolated linearly from nodal values of the flow mesh [26]. This Lagrangian numerical method is chosen for a variety of reasons, including (i) it allows for easy and effective calculation of the spatial moments of the plume and (ii) given the periodic nature of the flow domain it makes it possible to consider transport through a very large number of unit cells without requiring a prohibitively large numerical mesh. Specifically a particle can have any $(x, y)$ location, which is simply stored as a vector. It can have arbitrarily large $x$ and its velocity, needed to calculate its next spatial location, can be calculated for this location by simply noting that it is identical to the velocity at location $\bmod (x, L)$, where mod denotes the remainder function, due to the channel's periodic structure.

\subsection{Effective transport Model - Spatial Markov Model}

One of the primary outcomes of Taylor dispersion is that at asymptotic times a two- or three-dimensional transport process is properly described by a one-dimensional transport equation in the longitudinal direction of flow. In this study we aim to develop an effective one dimensional transport equation that is valid at pre-asymptotic times. To this end we will build our effective model using a spatial Markov framework. Like the classical random walk described above, spatial Markov models break the solute plume into a large, in principle infinite, number of particles. At a step $n$ each particle has a position $x_{i}^{(n)}$ and travel time $t_{i}^{(n)}$, which can be grouped into vectors $\left\{\mathbf{x}^{(n)}\right\}$ and $\left\{\mathbf{t}^{(n)}\right\}$ describing the kinematic state of the particle population at step $n$. The governing equation for transport can be written as 


$$
\begin{aligned}
& \mathbf{x}^{(n+1)}=\mathbf{x}^{(n)}+\Delta \mathbf{x}^{(n)} \\
& \mathbf{t}^{(n+1)}=\mathbf{t}^{(n)}+\Delta \mathbf{t}^{(n)} .
\end{aligned}
$$

$\Delta \mathbf{x}^{(n)}$ and $\Delta \mathbf{t}^{(n)}$ are successive spatial and temporal increments. The spatial Markov model is based in the hypothesis that Lagrangian velocities form a Markov process in space. Physically one can justify this property for our setup from the following argument. The transition time distribution that a particle will have to cross one pore depends solely on which streamline it enters the periodic domain on. Likewise the probability of which streamline one starts the next jump on, and thus the probability of the next transition time, depends also on the streamline where it entered. Thus the Markov property arises quite naturally for this system. In principle $\Delta t$ and $\Delta x$ can be random with joint distribution $\psi(x, t)$; independent [8] and coupled cases [24, 39] have been also considered. In many studies to date a common approach is to fix $\Delta x$ to a constant value such that $\psi(x, t)=\psi(t) \delta(x-\Delta x)$; in this particular case the most logical choice would be the unit cell length, i.e. $\Delta x=L$. Then the travel time distribution describes the distribution of times it takes particles to traverse one 'pore'. It has to date also been common to take the $\Delta t$ as independent identically distributed variables [8], but a series of recent papers [13, 14, 38] have shown that it may be important to account for the fact that successive time steps can be correlated, particularly if one wishes to capture transport at pre-asymptotic times well. Physically this reflects the fact that particles that traverse a pore quickly have a higher probability of traversing the next one quickly too and vice versa because fast particles will tend to stay on faster streamlines and slow particles on slower streamlines until diffusion smears this effect out. This conceptual picture is valid for high Péclet numbers. In order to develop this effective model, we use the fine scale simulations from sections 2.2 and 2.3 to build the distribution for $\Delta t$ and quantify the correlation between successive steps. 


\subsection{Metrics and Observables}

Here we define the metrics and observables that we will use to understand the system of interest and assess the effective spatial Markov model.

\subsubsection{Longitudinal Spatial Moments}

Two classic metrics of solute transport are the first and second centered spatial moments of the plume. Respectively these are given by

$$
m_{1}=\int_{-\infty}^{\infty} \int_{-h(x)}^{h(x)} x C(x, y, t) d y d x
$$

and

$$
\kappa^{2}(t)=\int_{-\infty}^{\infty} \int_{-h(x)}^{h(x)} x^{2} C(x, y, t) d y d x-m_{1}^{2}
$$

The first spatial moment $m_{1}(t)$ represents the horizontal position of the center of mass of the plume and is a measure of the longitudinal position of a plume. $\kappa^{2}$ is the second centered moment, which represents the spatial variance of the plume and is a measure of the spreading around the center of mass. This is closely related to the effective dispersion coefficient since

$$
D^{e}(t)=\frac{1}{2} \frac{d \kappa^{2}}{d t}
$$

which in the limit $t \rightarrow \infty$ tends to the asymptotic constant Taylor dispersion coefficient. At early times it will tend to evolve superdiffusively with time.

\subsubsection{Unit Cell Breakthrough Curves}

One of the key components of any random walk model is the distribution of travel times $\psi(\Delta t)$. For $\Delta x=L$ as we have defined it for our spatial Markov model, this distribution can be obtained by measuring the first passage time distribution of particles at $x=x_{0}+L$ where $x_{0}$ is the starting $x$ position of the particles. This is qualitatively similar to the breakthrough curve at this location, although caution must be taking in noting differences between breakthrough curves and first passage times (e.g. [2]). The wider this distribution, the wider 
the range of arrival times between fast and slow moving particles, leading to potentially greater dispersive spreading.

\subsubsection{Transition Matrix}

In order to account for the correlation between successive time steps, we must find the conditional probability density $r\left(\Delta t \mid \Delta t^{\prime}\right)$, which characterizes the probability of having a time increment $\Delta t$ given that the previous time step was $\Delta t^{\prime}$. If correlation were non-existent in the system, the conditional probability would be independent of $\Delta t^{\prime}$ and the conditional distribution would equal the distribution of travel times: $r\left(\Delta t \mid \Delta t^{\prime}\right)=\psi(\Delta t)$. When correlation is important this is not true. Quantifying this correlation formally is not numerically straightforward and so we adopt a discrete approach introduced by [13].

Let us discretize the time step distribution $\psi(\Delta t)$ into a finite number $n$ of discrete classes in the range $C_{i}(1 \leq i \leq n)$ such that a particle belongs to bin $C_{i}$ if its transition time lies in the range $\Delta t_{i} \leq \Delta t<\Delta t_{i+1}$. In this work the discretization is performed such that each class is equiprobable. For such discretization, the structure of the transition matrices is due to correlation effects only and is not influenced by distribution effects. Alternative discretizations that have been explored previously include linear in time or linear in log time. The latter is particularly interesting to refine the transitions that occur in the tails. For simulation purposes these discretization work well but they do not allow for as simple a physical interpretation of the transition matrices as the present one since the value of individual transition probabilities depends both on the strength of the correlation and on the probability of occurrence of each class.

Now, given these classes, we can discretize $r\left(\Delta t \mid \Delta t^{\prime}\right)$ into a transition probability matrix that provides the probability of jumping between classes between successive time steps. The transition matrix is defined as

$$
T_{i j}=\frac{\int_{\Delta t_{i}}^{\Delta t_{j+1}} \int_{\Delta t_{i}}^{\Delta t_{i+1}} r\left(t \mid t^{\prime}\right) \psi\left(t^{\prime}\right) d t^{\prime} d t}{\int_{\Delta t_{i}}^{\Delta t_{i+1}} \psi(t) d t}
$$


$T_{i j}$ characterizes the probability for a particle to jump from class $i$ in one step to class $j$ in its next step. $T_{i j}$ is calculated numerically by tracking how long it takes a particle to traverse a first pore as well as how long it takes to traverse the next one. The travel time to cross the first pore is used to assign its $i$ class and the travel time across the second pore its $j$ class. This process is repeated for a sufficiently large number of particles to populate $T_{i j}$. In this study one million particles were used. An important question is how many classes are adequate to resolve the pertinent dynamics. In a previous work ([38]), it was shown that reliable effective models can generally be built with as few as 10 classes, although in this work we used a larger number of 25 .

A novel metric that we introduce here is

$$
I C=\frac{1}{N_{c}} \sum_{i=1}^{N_{c}} \sum_{j=1}^{N_{c}} T_{i j}^{2},
$$

where $N_{c}$ is the number of discrete velocity classes. $I C$ quantifies how important correlation in a given system is. It is bounded between $1 / N_{c} \leq I C \leq 1$. If there is no correlation between successive steps each entry in the transition matrix will be identical and equal to $T_{i j}=1 / N_{c}$ and $I C=1 / N_{c}$. If the system is perfectly correlated (i.e. each successive step is identical to the last) then the diagonal elements of the correlation matrix will be $T_{i i}=1$ and all others zero such that $I C=1$.

\section{Results}

For all results presented here $\bar{u}=1,2 \bar{h}=1$ and $\nu$ and $D$ are adjusted accordingly to obtain the correct values of $R e$ and $P e$. All time units in this work correspond to a characteristic advection time, defined as the time it would take a particle moving at mean velocity $\bar{u}$ to traverse distance $2 \bar{h}$.

\subsection{Breakthrough Curves and Transition Matrices}

Unit cell breakthrough curves for $P e=100$ and $P e=1000$ are shown in figure 3 . In all cases a flux weighted pulse injection was performed, injecting a 
line at the narrowest part of the pore throat, i.e. $x_{0}=0$ in equation (1). The associated transition matrices are depicted in figure 4 . The same color scale is used for all transition matrices and both values of $P e$ to highlight similarities and differences.

At the lowest Reynolds number, $R e=1$, where recirculation zones are nonexistent, the breakthrough curves look very similar to those that have been published in previous studies $[13,14,38]$. The breakthrough curves have an early arrival of concentration that causes a rapid rise of the breakthrough curve up to a peak. After the peak the concentration decreases monotonically with time. However as the Reynolds number increases and the recirculation zones occupy a larger and larger fraction of the unit cell, the post-peak part of the curve does not decrease monotonically; there is a double-hump, which becomes more distinct as Re increases. Such double peaks have not been observed in previous studies $[13,14,38]$. The dominant and distinct feature of the flows studied here compared to those of previous studies is the presence of large recirculation zones. $[13,14]$ studied transport in highly heterogeneous porous media at the continuum scale, for which flow is governed by Darcy's law, which does not mathematically permit the existence of recirculation due to the fact that pressure must be monotonically decreasing in the direction of flow along a streamline, precluding streamlines from wrapping back on themselves. This restriction does not exist for Stokes or Navier-Stokes flow, where recirculation zones can and often do occur. [38] studied flow in a geometry similar to the present one, but at small Reynolds numbers, where any recirculation zones that do exist cover a rather small area and exhibit very small fluid velocities, which renders them practically equivalent to zones of immobile fluid. Based on this we initially hypothesized that the presence of this double peak is clearly associated with recirculation. Physically we believed it is a reflection of the fact that a particle can enter a recirculation at one point and exit at an upstream point from where it entered, which can result in the double-hump feature.

Another notable feature is that as the size of the recirculation zones increases the distribution of travel times becomes broader with earlier arrival times as $R e$ 
increases and later tails for cases with large recirculation. Physically this reflects the fact that the recirculation zones cause a faster flow speed through the central channel as well as trapping and delays in breakthrough. In the breakthrough curves the primary role of Péclet number appears to be a sharper initial peak and greater late time tailing, which is consistent with previous observations. This reflects the fact that with weaker diffusive effects more of the solute remains in the main channel breaking through quickly, but that the solute that does enter the recirculation zones will reside there for longer.

We also measured the amount of time that the particles spend in the recirculation zone and the mean times are approximately $t=11$ for both Reynolds numbers for the $P e=1000$ case and approximately $t=2.8$ for the $P e=100$ cases. The similarity for both $R e$ numbers at each $P e$ helps explain why the late time tails for the breakthrough curves at each $P e$, once recirculation exists, in figure 3 are so close regardless of $R e$.

The transition matrices in figure 4 display many of the features observed in previous studies. All of the matrices have a diagonal dominance reflecting the fact that a particle's most probable next transit time sits in the same class as its previous one. With the chosen color scale this is difficult to see for the $P e=100$ cases, but it becomes clearer if one rescales the color scale. The specific color scale in figure 4 does not aim at showing this, but rather at highlighting contrasts and the weakness of correlation in the $P e=100$ cases relative to the $P e=1000$ ones. The strongest correlation exists for the greatest and lowest transit times; i.e. particles in the center of the channel with the highest velocities will continue to sit there and particles with the slowest velocities, which are those that enter the recirculation zones, are most likely to enter the next recirculation zone. This is visually depicted in figure 5 and discussed in greater detail in section 4.2. It appears that the structure of the matrices, at least qualitatively, is weakly sensitive to Reynolds number. However, the dependence on the Péclet number is clearly evident. For the $P e=100$ case the greatest values in the transition matrix are approximately 0.06 , while for the $P e=1000$ case they are 3 times greater. If there were absolutely no correlation all values in the transition matrix 
would be equal to 0.04 . This suggests that correlation effects for the $P e=100$ case may not be all that important, while they may play a significant role in the $P e=1000$ case. Again, this will be discussed in greater detail below.
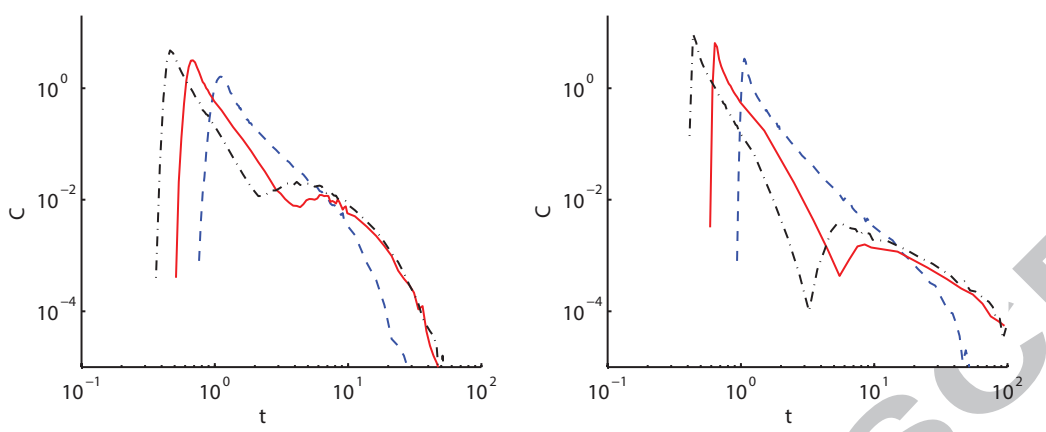

Figure 3: Breakthrough curves after a single unit cell for $R e=1$ (blue - -), 30 (red -) and 100 (black -.) at $P e=100$ (left) and $P e=1000$ (right).

\subsection{Role of Recirculation Advection in Immobile Zones}

As noted above, the recirculation zones change the nature of the single unit breakthrough curves by increasing the breadth of travel time distributions and allowing the emergence of a double hump. In order to test the true influence of advective recirculation in these regions we reran the transport simulations, but under the condition where all velocities in the immobile regions are set to 0 and particles can only move by diffusion. Advection only occurs in the main channel flow and any transport in the 'recirculation' zones is purely diffusive. Note that this is unphysical as the flow will no longer satisfy the Navier-Stokes equation and is purely done as a numerical experiment to isolate the role of advection in the recirculation zones.

The unit cell breakthrough curves for cases with recirculation turned on and off are shown in figure 6 . Results are shown for the $R e=30$ and 100 cases as no recirculation exists at $R e=1$. As we had originally hypothesized, for the $P e=100$ case the double-hump feature does indeed disappear and the post peak breakthrough curve decreases monotonically when advection is turned off in the recirculation zones. However for the $P e=1000$ case a double hump does 

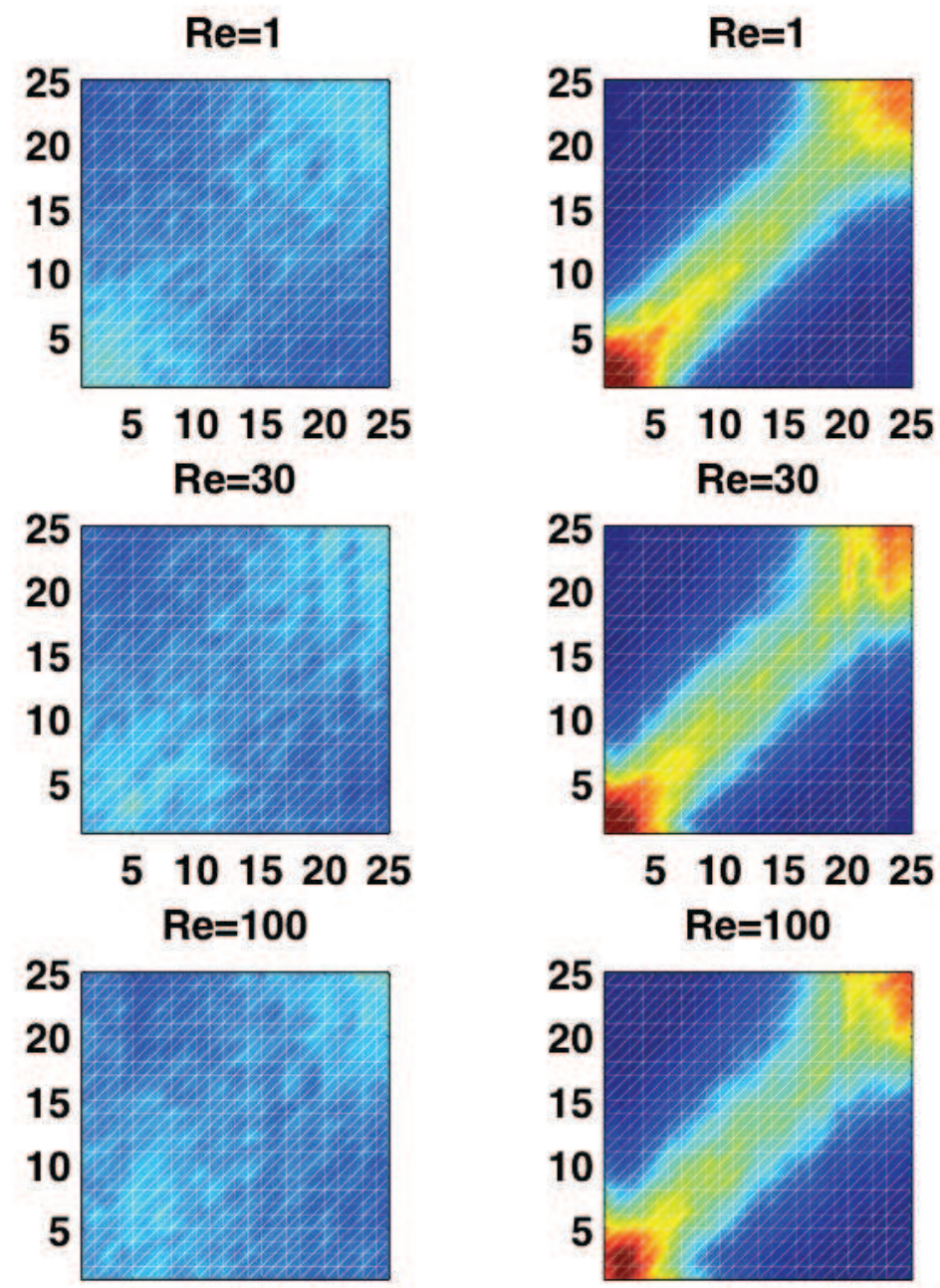

510152025

510152025

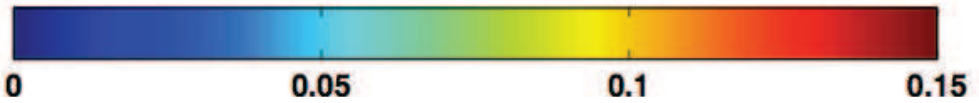

Figure 4: Spatial transition matrices T corresponding to different Reynolds numbers for $P e=$ 100 (left column) and $P e=1000$ (right column). The horizontal axis represents the initial transit time class and the vertical axis represents the next transit time class. Small matrix indices correspond to small Lagrangian velocities (large transit times), and large matrix indices correspond to large Lagrangian velocities (small transit times). The color scale represents the transit time transition probabilities along particle paths. 


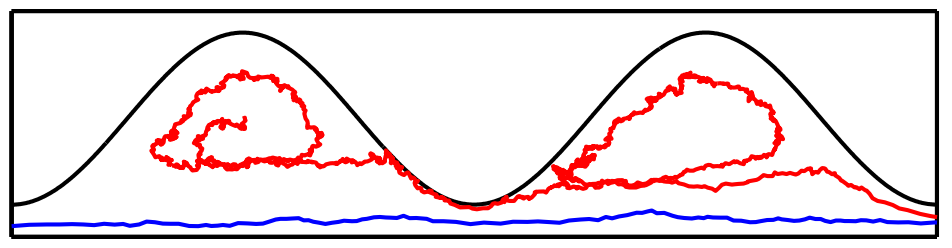

Figure 5: Particle Trajectories for two sample particles at $R e=30$ and $P e=1000$. The blue one starts in the fast channel and persists there. The red one starts in an immobile zone and is also trapped in the next one. This visually demonstrates strong correlations between successively fast and successively slow transition times .

still occur for both Reynolds numbers, but it occurs at lower concentrations and later times, still suggesting that advection in the recirculation zones is a dominant contributor to this effect. Physically, the proposed mechanism for the double hump, by which a particle enters the recirculation region at a one point and exits at an upstream point can still occur when motion is purely diffusive; its occurrence is just less probable, hence the shift of the second hump to later times and lower concentrations.

We also conducted simulations where we maintained zero velocity and also turned horizontal diffusion off in the recirculation zones, allowing particles to only diffuse vertically. In all of these curves the second hump is not discernible. Other than this the breakthrough curves look virtually unchanged. This provides some evidence that it is indeed horizontal transport mechanisms in the recirculation zones that are responsible for the emergence of this double hump behavior.

While as part of this discussion we have focused heavily on the difference between the advective and diffusive immobile zones with regard to the doublehump, it is important to note that this feature occurs at intermediate breakthrough times. An equally interesting observation is that both the early and late arrival times seem to be virtually unaffected by the presence of advection in the recirculation regions. In many cases early and late arrival times are those of greatest practical concern [e.g. 11], suggesting that perhaps resolving these 
recirculation zones is not critical depending on a specific application. From the scientific perspective we find these differences interesting and important as they are signatures one can clearly look for in real breakthrough curves, providing additional interpretation possibilities of observations. While not depicted here, the transition matrices for purely diffusive recirculation zones are virtually identical to those with advection turned on.
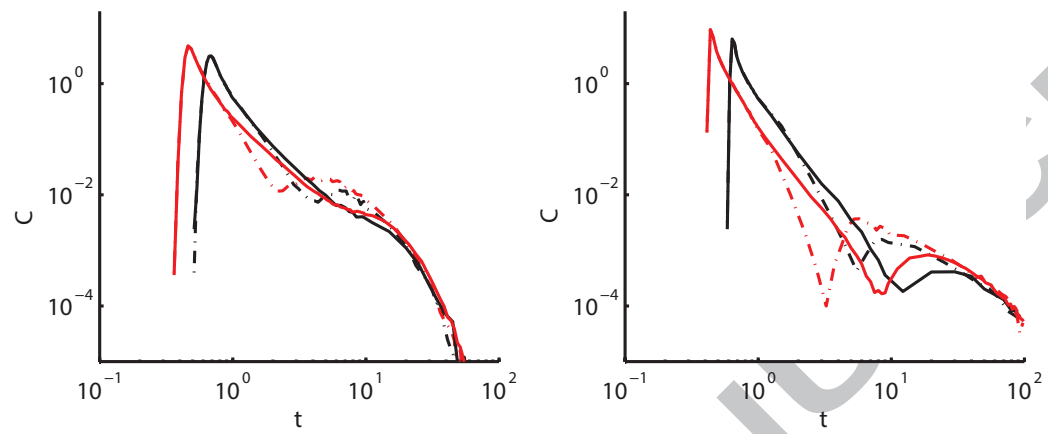

Figure 6: Breakthrough Curves for $R e=30$ (red) and 100 (black) for $P e=100$ (left) and $P e=1000$ (right). The dashed lines represent simulations where the recirculation zones are treated as inertial advective zone and resolve the velocity field while the solid lines represent simulations where advective effects in the recirculation zones are neglected .

\subsection{Down Stream Breakthrough Curves}

While studying the unit cell breakthrough curve is key to developing our effective modeling framework, ultimately the goal is to understand how transport behaves at greater downstream distances. Figure 7 displays breakthrough curves at greater distances $(x=5 L, 10 L, 20 L$ and $50 L)$, which will be used to test the predictive capacity of the effective spatial Markov model. To supplement the discussion of the previous section, breakthrough curves are shown for cases where advection in the recirculation zones is both switched on and off.

In all cases the early breakthrough curves preserve many of the signatures of the single cell breakthrough curves, displaying features like tailing and the double hump, associated with the presence of recirculation zones discussed above. For the $P e=1000$ curves many of these features are preserved in all breakthrough curves, while for the $\mathrm{Pe}=100$ the breakthrough curves at large distances 
(e.g. $x=50 L$ ) appear more Fickian, which is consistent with the idea that at these late times transport has converged to an asymptotic Fickian Taylor regime. Similar differences, such as smaller second humps but similar early arrivals and late time tails, exist as between the curves where advection is left on and turned off in the recirculation zones as was observed for single cell breakthrough curve.
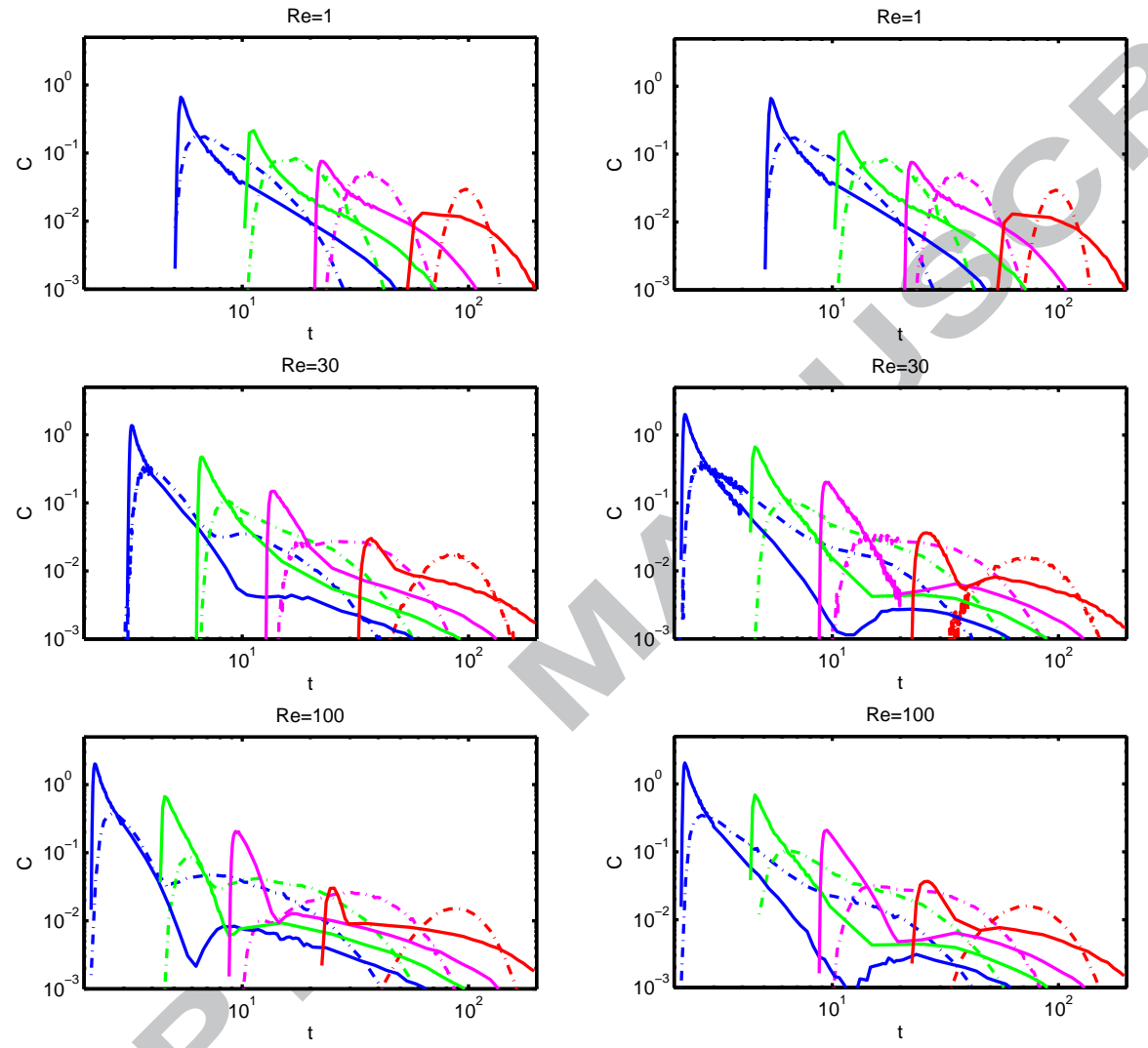

Figure 7: Breakthrough Curves for $R e=1,30$ and 100 for $P e=100$ (dashed -) and $P e=1000$ (solid -) at distances of $x=5 L$ (blue), $10 L$ (green), $20 L$ (purple) and $50 L$ (red). The left hand column represents models where we treat the recirculation zones as advective (Navier-Stokes flow), while on the right hand side we treat recirculation zones as purely diffusive . 


\subsection{Predictive Capacity of Spatial Markov Model}

One of the primary goals of this work is to test the effective spatial Markov model, which is developed with the single unit breakthrough curves and transition matrices. In order to test it we consider two measures. First we test the effective model's ability to predict the late time breakthrough curves discussed in section 3.3. It is common to find fits to a single breakthrough curve as a measure of an effective model's success, although multiple examples of predictions of concentration at various times and downstream locations do also exist $[7,8]$. We argue that full model validation should require this prediction of other breakthrough curves with no additional fitting. The second measure is the model's ability to reproduce the second centered moment of the plume, which is another traditional measure of a model's strength. The work of Dentz \& Bolster [25] highlights why there is a need for cross-validation using breakthrough curves and spatial moments.

\subsubsection{Comparison of Down Stream Breakthrough Curves}

Predictions with both an uncorrelated and correlated spatial Markov model for $P e=100$ and $P e=1000$ cases are shown in figures 8 and 9. The uncorrelated effective model takes the unit cell breakthrough curve as the probability distribution for transit times, but does not use information from the transition matrix to condition a transit time on its previous value - i.e. successive steps are completely independent of one another, while the correlated spatial Markov model imposes correlation of successive steps using the transition matrix.

For the $P e=100$ case both models do an excellent job of predicting the late time breakthrough curves, including early arrivals, intermediate effects such

as the double hump and any late time tails. For the $P e=1000$ case the correlated spatial Markov model does an equally good job of predicting the measured breakthrough curves. However, the uncorrelated model is unable to reproduce certain features observed in the simulated curves. In particular it does not faithfully reproduce early and intermediate time arrivals, underestimating the peak concentration of the breakthrough curves. It appears, at least for 
the $R e=30$ and 100 cases to capture the late time tails. This suggests that correlation of fast moving particles is particularly important as these are the main contributors to the early arrival times.

The fact that correlation in the effective model is unimportant for $P e=100$ is perhaps unsurprising given how weak correlation effects are in the transition matrices for these cases. However, it should be clear that correlation becomes increasingly important as $P e$ increases. Again this comes as no surprise as it is diffusion that allows particles to jump across streamlines and thus transition between velocity classes.

These results demonstrate a very important point though. If correlation effects are not important the unit cell breakthrough curve provides sufficient information to predict late time behavior of the plume and traditional effective models such as CTRW characterized by uncorrelated transition times., multi-rate mass transfer (MRMT) and fractional advection dispersion equations (fADE) $[8,30,20,7,6]$ should work well in a predictive sense. If however, correlation effects are important, they must be incorporated into the model. It is interesting to note that incorporating correlation effects appears to play its most important role in predicting early concentration arrivals as to date much focus on effective models has been aimed at capturing late time anomalous tails.

\subsubsection{Moments}

Figure 10 displays the evolution of the second centered moment against time for three different Reynolds numbers $R e=10,30$ and 100. The second centered moment is larger for larger Re, which is consistent with the observations of [15]. To address how well our models work at capturing the observed simulated second centered moments we introduce the following measure of error

$$
E r=\frac{1}{N} \sum_{i=1}^{N} \frac{\left|O_{i}-M_{i}\right|}{O_{i}},
$$

where $O_{i}$ is observed data from the fine scale simulations and $M_{i}$ is model data from the spatial Markov and uncorrelated CTRW models. This is a measure of 

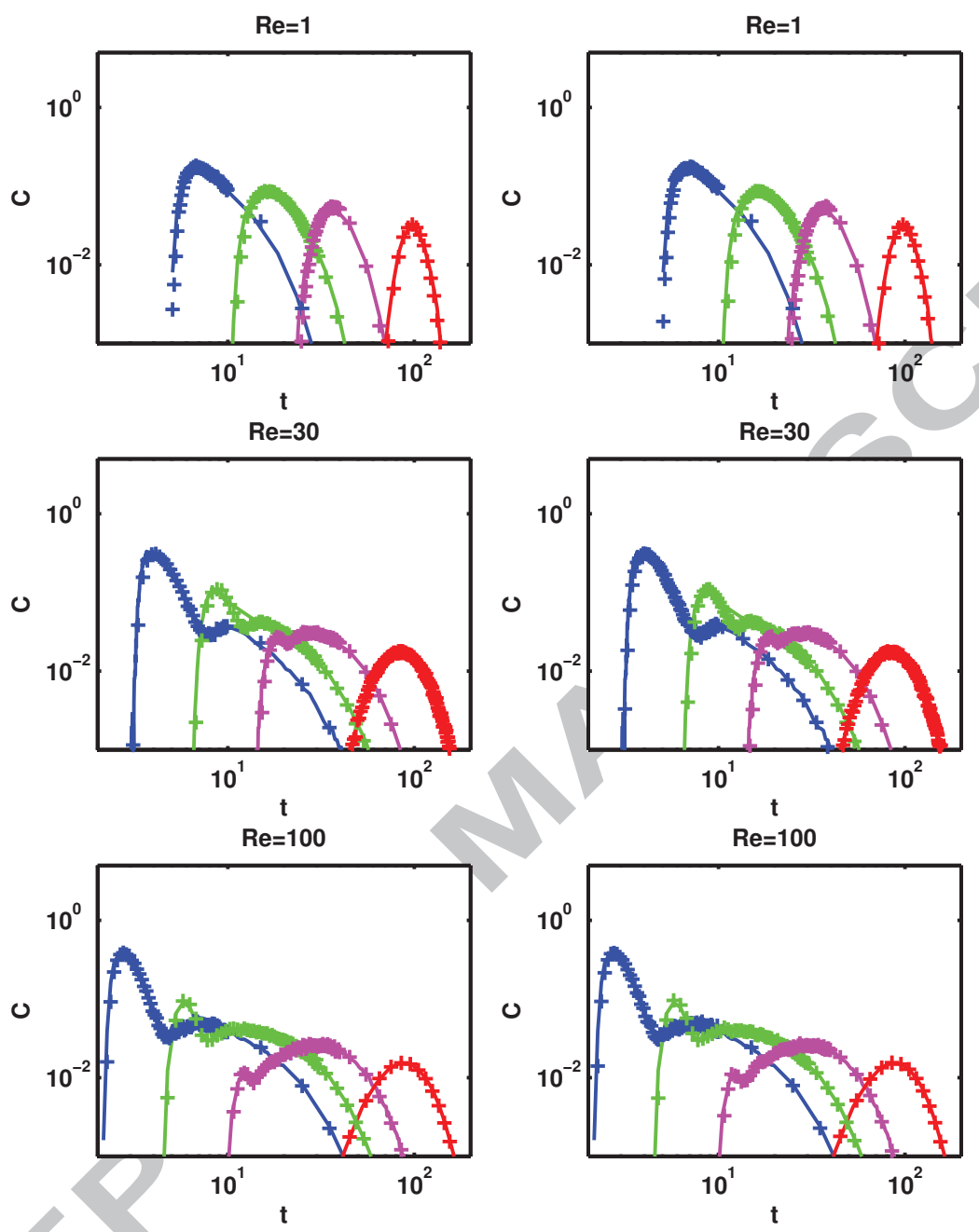

Figure 8: Comparison of Breakthrough Curves for $R e=1,30$ and 100 for $P e=100$ at distances of $x=5 L$ (blue), $10 L$ (green), $20 L$ (purple) and $50 L$ (red). Solid lines represent simulated results and symbols are effective model results. The left hand column represents models with no correlation between successive steps, while the right hand column represents the spatial Markov model with correlation between successive steps 

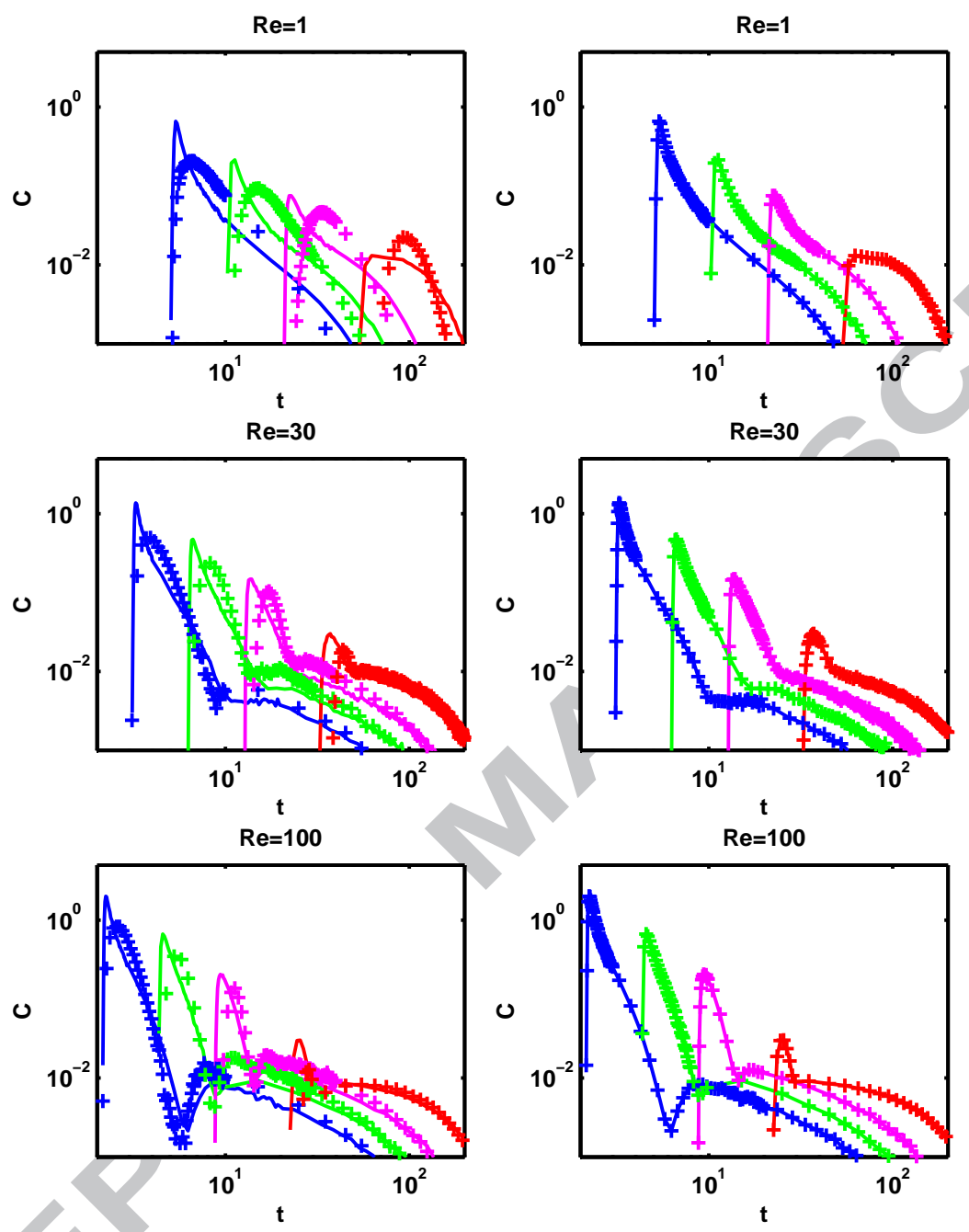

Figure 9: Comparison of Breakthrough Curves for $R e=1,30$ and 100 for $P e=1000$ at distances of $x=5 L$ (blue), $10 L$ (green), $20 L$ (purple) and $50 L$ (red). Solid lines represent simulated results and symbols effective model results. The left hand column represents models with no correlation between successive steps, while the right hand column represents the spatial Markov model with correlation between successive steps 
by what fraction the modeled results are off from the simulated observed results. For $P e=100 E r$ is less than $5 \times 10^{-3}$ for the correlated model and less than $2.5 \times 10^{-2}$ for the uncorrelated model. Both errors can be considered small, but clearly the correlated model outperforms the uncorrelated one demonstrating that even for this $P e$ some small correlation effects are present and may be necessary depending on what one is interested in. For the $P e=1000$ case Er is around $1.5 \times 10^{-2}$ for the correlated model and 0.28 for the uncorrelated model, demonstrating a significant improvement with the inclusion of correlation. This is consistent with the BTC predictions, where the uncorrelated model failed to adequately capture the peak early arrivals, which would lead to a greater spread in mass around the plume's mean position. Again it is clear that there is a transition from correlation being unimportant to important, which we discuss in further detail in the next section.

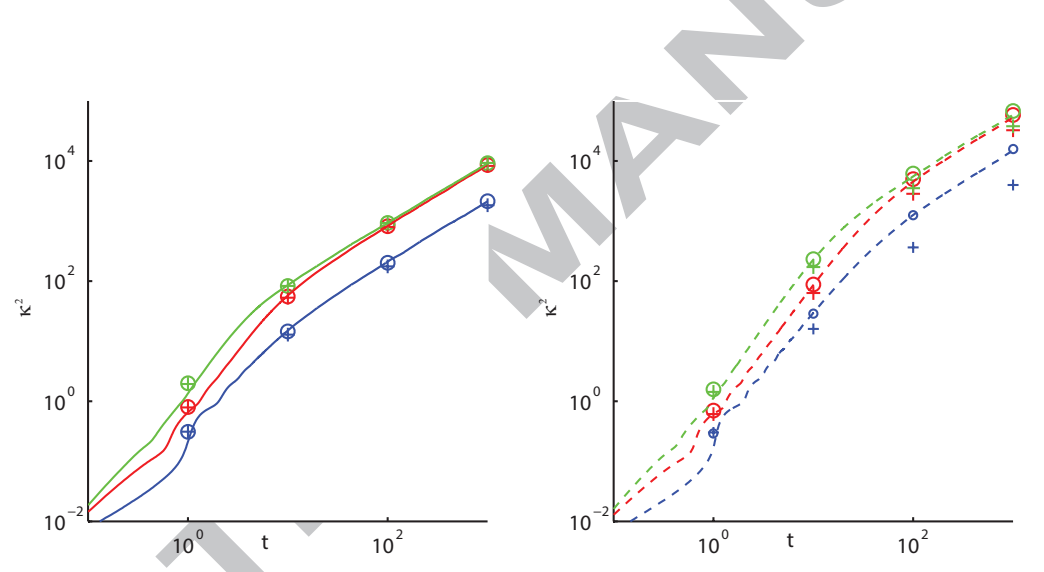

Figure 10: A comparison of the second centered moment between simulations (solid lines) and spatial Markov (o) and uncorrelated (+) predictions. On the left are cases for $P e=100$ and on the right $P e=1000 . R e=1$ (blue), 30 (red) and 100(green) are shown.

\subsection{The Importance of Accounting for Correlation in Successive Jumps}

As noted above it seems clear that there is a transition from correlation being unimportant to correlation being important in developing the effective CTRW model somewhere between $P e=100$ and $P e=1000$. In order to fully understand this let us take a look at the importance of correlation parameter 
$I C$ defined in equation (12). A plot of how $I C$ varies with $P e$ is shown in figure 11. For $P e<100$ it is clear that $I C$ is close to its absolute minimum value, which is $1 / 25$. This suggests that for these values of $P e$ the transition matrix is relatively homogenous, meaning that there is little correlation between successive jumps. For $P e>100$ there is a monotonic increase of $I C$ with $P e$ emphasizing that correlation effects become more and more important as $P e$ increases. Interestingly for the $P e=1000$ case where we have already shown that correlation effects must be included to reliably reproduce observables, $I C=$ 0.07 , which one could argue is not all that large. While $I C$ clearly measures some degree of correlation effects, better metrics should be sought. This demonstrates perhaps how strong the influence of correlation, even if relatively weak, truly can be. Some of the large $P e$ numbers shown in figure 11 may seem very large for $R e=100$. However they may not be unusual as for flowing water systems $S c=$ $O(100-1000)$, while for flowing gasses $S c=O(1)$ may be more typical. Results for Péclet numbers much larger than $10^{5}$ may not be entirely physical for water and should be taken with a grain of salt, although they may apply for example for solutes in more more viscous fluids (e.g. oil, glycerol, molasses). Additionally materials that diffuse more slowly, such as larger nanoparticles, which can have diffusion coefficients several orders of magnitude smaller than typical solutes [34], may exhibit higher Pe, although in this case further physics may be required to model the system adequately. However, it should be noted that this plot merely serves to demonstrate the increasing importance of correlation with $P e$. To demonstrate that the spatial Markov model is able to replicate results even at these larger numbers a comparison of late time breakthrough curves for $R e=$ 100 and $P e=10^{5}$ is shown in figure 12. Again, note the correlated model is able to capture the early time peaks exactly, while the uncorrelated one fails. At this large Péclet number the uncorrelated model also fails to capture the later time tails. 


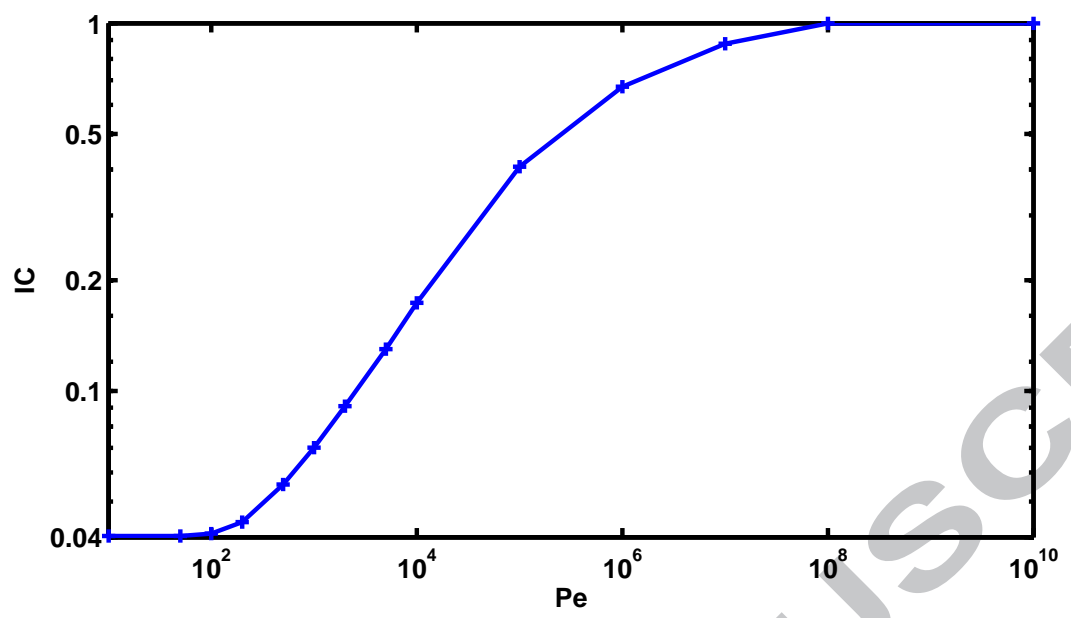

Figure 11: A plot of the Importance of Correlation Parameter $(I C)$ against Péclet number for $R e=100$.
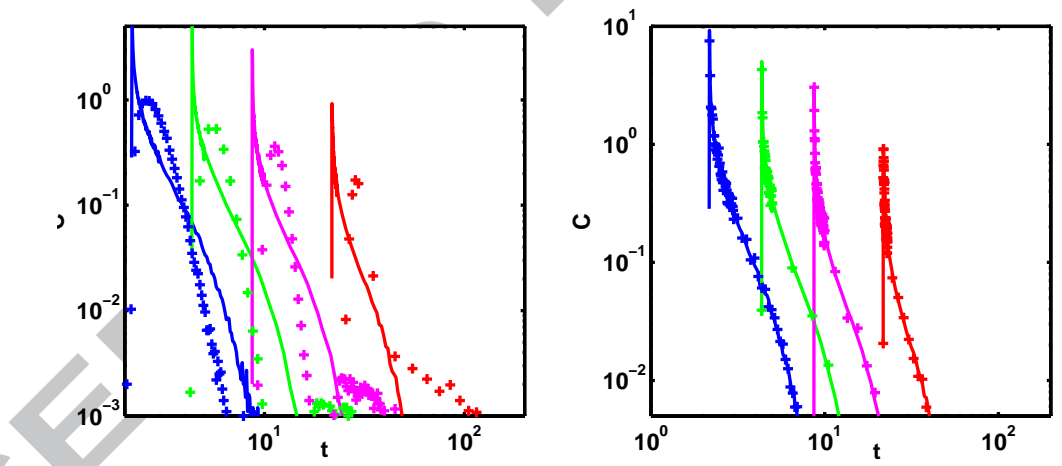

Figure 12: Comparison of late time breakthrough curves at distances of $x=5 \mathrm{~L}$ (blue), $10 \mathrm{~L}$ (green), $20 L$ (purple) and $50 L$ (red) for the uncorrelated (left) and spatial Markov model model (right) for $R e=100$ and $P e=10^{5}$, which would correspond to a solute with Schmidt number 1000, an order of magnitude typical for many solutes in aqueous environments. Solid lines are measured from simulations and symbols are model results. 


\section{Discussion}

\subsection{Asymptotic Dispersion}

In this section we discuss and attempt to explain physically, using the models and results presented so far, a to date unexplained observation made by [15]. As outlined in the introduction, Bouquain et al. [15] observed that as the Reynolds number increases, for fixed $P e$, there is an increase in the asymptotic effective dispersion coefficient. As the area of the recirculation zones grows there is a strong and rapid growth of asymptotic dispersion coefficient with Reynolds number that eventually almost saturates to some upper value. The rapid growth at intermediate Reynolds number appears to be related to the growth of the area of the recirculation zone as a function of the Reynolds number. Note that this behavior is observed at fixed $P e$. In a physical experiment, increasing the mean flow velocity to increase the Reynolds number would also increase the Péclet number, hence the behavior as a function of Reynolds alone is difficult to investigate experimentally. As mentioned in the introduction, [15] have already shown that the dependence of the asymptotic dispersion coefficient on Reynolds and Péclet can be separated into two factors, the factor dependent on Péclet being a power law with a negative exponent.

To understand this behavior let us look at the expected theoretical behavior of asymptotic dispersion coefficients in continuous time random walks, which are analogous to our uncorrelated model. In a seminal paper [43] showed that the second centered moment of a plume moving with a CTRW is given by

$$
\begin{array}{r}
\sigma^{2}(t)=\overline{l^{2}} \mathcal{L}^{-1}\left(\frac{\psi^{*}(s)}{s\left(1-\psi^{*}(s)\right.}\right)+\bar{l}^{2} \mathcal{L}^{-1}\left(\frac{2 \psi^{* 2}(s)}{s\left(1-\psi^{*}(s)^{2}\right.}\right) \\
-\bar{l}^{2}\left[\mathcal{L}^{-1}\left(\frac{\psi^{*}(s)}{s\left(1-\psi^{*}(s)\right.}\right)\right]^{2},
\end{array}
$$

where $\mathcal{L}$ is the Laplace transform operator, $\mathcal{L}^{-1}$ the inverse Laplace transform operator, $s$ the Laplace domain variable, $\psi^{*}(s)$, the Laplace transform of the travel time distribution, $\bar{l}$ the mean jump distance and $\overline{l^{2}}$ the mean of the jump distance squared. For our case $l$ is constant and equal to the length of the pore. 
Recall that late time behaviour (i.e. $t \rightarrow \infty$ ) in the time domain corresponds to small Laplace domain variable (i.e. $s \rightarrow 0$ ). If $\psi(t)$ has finite first and second moments, which in all our cases it does [43] argued that for small $s$, $\psi^{*}(s) \sim 1-s \bar{t}+\frac{1}{2} s^{2} \overline{t^{2}}+\mathcal{O}\left(\overline{t^{3}}\right) ; \bar{t}$ and $\overline{t^{2}}$ are the first and second moment of the travel time distribution respectively, which are calculated from the first passage times obtained in the numerical simulations. Substituting this expression into (14), expanding in small $s$ and performing the inverse Laplace transforms it can be shown that at large times to leading order in time, its time dependence can be written as

$$
\sigma^{2}(t)=\bar{l}^{2}\left(\frac{1}{\bar{t}}+\frac{2}{\bar{t}}\left[\frac{1}{2} \frac{\overline{t^{2}}}{\bar{t}^{2}}-1\right]\right) t+O\left(t^{0}\right)
$$

which scales linearly in time, with all other order terms of order $O\left(t^{0}\right)$ or lower. For all cases considered the asymptotic mean velocity of the plume is equal to the mean velocity of the flow, meaning that $\bar{t}$ must be the same for all cases, since $\bar{u}$ is identical for all numerical simulations. Thus, since the asymptotic dispersion coefficient is equal to the time derivative of $\sigma^{2}(t)$

$$
D^{\infty}=\overline{l^{2}}\left(\overline{t^{2}}-1\right)
$$

The red circles in figure 13 correspond to predictions made with this formula for $P e=100$ and it can be seen that there is excellent agreement between this prediction and the values of the asymptotic dispersion coefficients measured from the random walk numerical simulations. Note that the observations made with numerical simulations correspond to times greater than the characteristic Taylor dispersion time. For this setup equation (16) in essence says that the asymptotic dispersion coefficient is directly proportional to the variance of the arrival time distribution. A look at the travel time distributions presented in figure 3 demonstrates why there is a large jump in asymptotic dispersion between the small and intermediate Reynolds numbers, but also why the value more or less saturates. As already mentioned previously as the Reynolds num- 


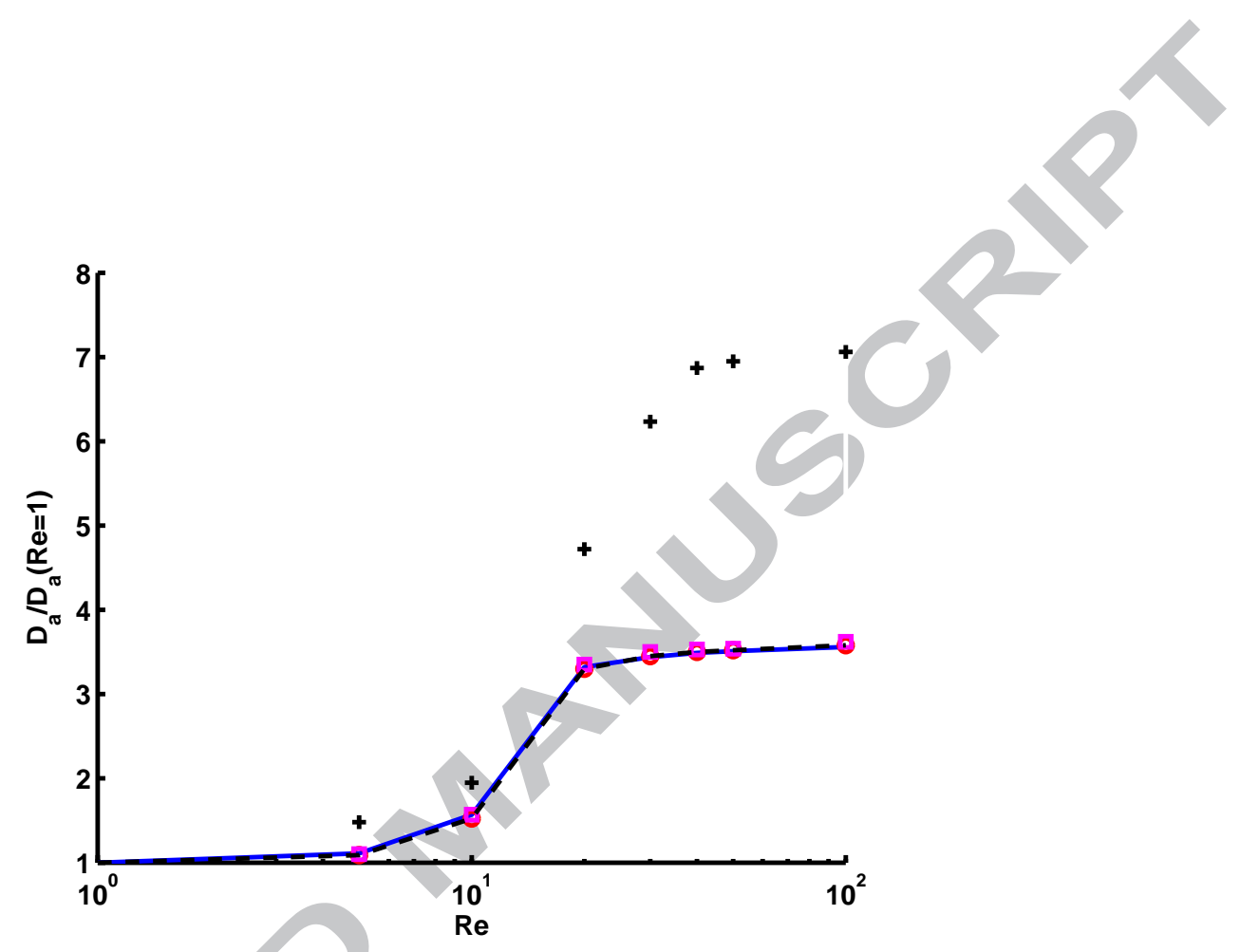

Figure 13: Asymptotic Dispersion Coefficient against Reynolds number for $\mathrm{Pe}=100$ and $\mathrm{Pe}=1000$. The dispersion coefficient is normalized by the value for $\mathrm{Re}=1$. The solid blue line $(\mathrm{Pe}=100)$ and dashed black line $(\mathrm{Pe}=1000)$ connect measured values from direct random walk simulations, while the red circles $(\mathrm{Pe}=100)$ and black plus signs $(\mathrm{Pe}=1000)$ correspond to values estimated using the uncorrelated CTRW prediction in equation. Finally the magenta square symbols correspond to the prediction for $\mathrm{Pe}=1000$ using the uncorrelated model with the transition time probability over a distance of $15 L$, rather than just $L$ (16) . 
ber increases and recirculation zones emerge there is a broadening of the arrival times with both greater earlier arrival as well as increased late time tailing. As the Reynolds number increases further the late time tail seems relatively unaffected, while there is a small shift in the early arrival times. The relative influence of this shift in early arrival times is small with respect to the variance and so the asymptotic dispersion coefficient as predicted by equation (16) changes very little. In essence it is the tails that control the asymptotic dispersion coefficient and once significant recirculation zones have formed these tails are almost identical for all Re.

If we apply equation (16) to the $P e=1000$ case as we did to the $P e=100$ case the resulting equivalent plot is also shown in figure 13. Note that the measured asymptotic dispersion coefficients behave almost identically to the $P e=100$ case, which is in line with the observations of [15], who showed that the ratio of asymptotic dispersion coefficients behaves the same regardless of Péclet number. However the results from applying (16) differ significantly from the measurements, overpredicting the ratio between asymptotic dispersion at $R e=100$ to $R e=1$ by about a factor of 2 . This is consistent with the ideas presented so far, because for $P e=1000$ correlation effects must be included and the theory leading to equation (16) does not account for correlations. We address this issue briefly in the next section.

\subsection{Probability of Successive Trapping}

A primary feature that arises in the considered flow as Reynolds number increases is the emergence and growth of the recirculation zone, which can be thought of as a trapping zone. Given the correlated nature of transport, particularly at larger Péclet numbers, a pertinent question is what is the probability of a particle being trapped in the next recirculation zone given that it has just exited a recirculation zone (see figure 5). This persistence of longer travel times is an important feature that the proposed spatial Markov model captures, but that an uncorrelated model would not capture as well; this explains for example why the late time tails are not captured by the uncorrelated model in figure 12 . 
Here we briefly discuss estimates of this probability of successive trapping.

Let us define $P_{t}$ as the probability for a particle to be trapped in the immediately next recirculation zone given that it has been trapped in a current recirculation zone. Thus

$$
P_{t}=p\left(t_{i+1}>t^{*} \mid t_{i}>t^{*}\right)
$$

where $t_{i}$ is the travel time of a particle through cell number $i$ and $t^{*}$ is the maximum such travel time that a particle can have without entering the recirculation zone. This can be estimated as the travel time along the streamline adjacent to the recirculation zone. Assuming that the trapping process is Markovian, the probability of $n$ successive trappings occurring along a particle's trajectory is simply $P_{t}^{n}$. This is because this probability is stationary (i.e. the probability of being trapped in recirculation zone 2 , given that the particle was trapped in recicrulation zone 1 is the same as being trapped in recirculation zone $n+1$ given being trapped in $n$ ). The average number of successive trapping events, $\langle n\rangle$, is then obtained as

$$
\langle n\rangle=\frac{1}{\sum_{n=1}^{\infty} P_{t}^{n}} \sum_{n=1}^{\infty} n P_{t}^{n}=\frac{P_{t}}{\sum_{n=1}^{\infty} P_{t}^{n}} \frac{d}{d\left(P_{t}\right)}\left(\sum_{n=1}^{\infty} P_{t}^{n}\right)
$$

where $\sum_{n=1}^{\infty} P_{t}^{n}$ is equal to $P_{t} /\left(1-P_{t}\right)$. Hence:

$$
\langle n\rangle=\left(1-P_{t}\right) \frac{1}{\left(1-P_{t}\right)^{2}}=\frac{1}{1-P_{t}}
$$

$P_{t}$ can be computed from the transition matrices or by direct numerical simulation, and, consequently, $\langle n\rangle$ using Eq. (19). Figure 14 shows how $P_{t}$ varies with Reynolds number. As the recirculation zones become larger and larger the probability of trapping increases, although the range of $P_{t}$ does not vary enormously, particularly for $P e=1000$. Interestingly for the larger Reynolds number cases the probability of successive trapping for the $P e=100$ case is larger than the $P e=1000$ case, while for the smaller Reynolds number $P_{t}$ is 
larger for $P e=1000$ : for the $P e=1000$ case the average number of successive trapping events $\langle n\rangle$ varies between 1.5 and 1.8 , while that for the $P e=100$ case varies between 1.4 and 2. This is due to the way diffusion and advection interact. Let us consider solute particles that have just exited a recirculation zone (by diffusion) and are therefore moving close to the boundary of the recirculation zone. Diffusion is what can allow them to enter the next recirculation zone; it is also what can allow them to move transversally away from the next recirculation zone. At lower Reynolds numbers, the latter process is enhanced by advection, as flow lines diverge significantly after going through the neck that separates consecutive cells; therefore increasing diffusion can actually decrease successive trapping. Conversely at larger Reynolds numbers the mainstream channel is quasi linear, and increased diffusion is observed to favor trapping in the recirculation zones over transport towards the center line of the cells.

Another interesting metric in thinking about correlation and successive trapping is, given that a particle is trapped in a recirculation zone what is the probability of it getting trapped in any downstream recirculation zone, i.e.

$$
p\left(t_{i+n}>t^{*} \mid t_{i}>t^{*}\right)
$$

It is important to recognize is that just because a particle is not trapped in the immediately next recirculation zone does not mean that it does not have a preferential trapping in further downstream ones as correlation effects can persist over several jump lengths. This metric provides an idea of how quickly the system decorrelates as eventually the probability of entering a recirculation zone should be independent of whether it was previously trapped or not. This metric provides a measure of memory in the system. Figure 15 (top) depicts this probability where initially all particles are trapped in recirculation zone 1 and then the probability of being trapped in downstream recirculation zones $2,3, \ldots 15$ is measured. Results for $\mathrm{Re}=30$ and 100 and $\mathrm{Pe}=100$ and 1000 are shown. It is immediately evident that for the $\mathrm{Pe}=100$ cases the probability of successive trapping is higher than the $\mathrm{Pe}=1000$ cases, reflecting the fact that 
with larger diffusive effects it is easier for particles to enter the recirculation zones. Additionally though for $\mathrm{Pe}=100$ the probability of entering any of the downstream recirculation zones is the same for all recirculation zones. This reflects the fact that all particles have almost equal access to all travel times as shown by the weak correlation and almost uniform transition matrices for the $P e=100$ cases. This is in effect why the uncorrelated model works as well as it does in reproducing observed metrics from the direct numerical simulations. For $P e=1000$ there is a decay in probability of trapping with downstream recirculation zone, reflecting the strong correlation.

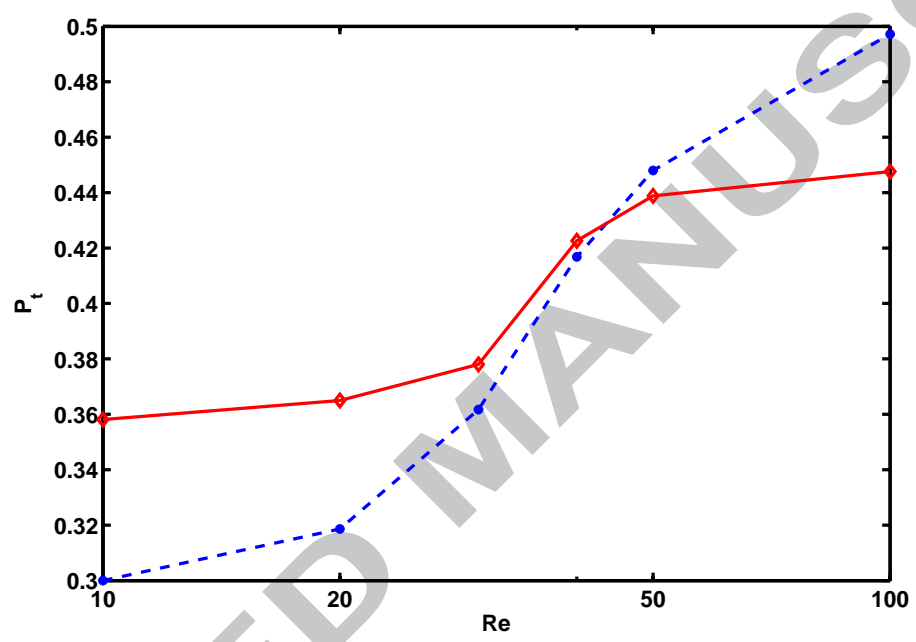

Figure 14: Probability of being trapped in the next recirculation zone given that a particle is trapped in the current one depending on Re. Red solid line corresponds to $\mathrm{Pe}=1000$ and the blue dashed line to $\mathrm{Pe}=100$.

An alternative manner of looking at this persistence of correlation is to look at the importance of correlation parameter $I C$ and the transition matrix. Due to the Markov property of the transition matrix one can look at correlations over multiple jumps by taking powers of the transition matrix

$$
T T(n)=T^{n}
$$


where $T T(n)_{i j}$ measures the transition probability after $n$ spatial jumps; i.e. it calculates the probability of a particle belonging to some class $j$, given that it belonged to class $i n$ jumps ago. Now we can calculate $I C$ for successive powers of $\mathrm{T}$, defined as the squared norm of matrix $T T(n)$ :

$$
I C_{T}(n)=\frac{1}{N_{c}} \sum_{i=1}^{N_{c}} \sum_{j=1}^{N_{c}}(T T(n))_{i j}^{2},
$$

A plot depicting $I C$ (normalized) against $n$, the number of steps, is shown in figure 15 (bottom). This appears to display the same quasi-exponential correlation structure as figure 15 (top). Thus the transition matrix combined with $I C$ provides all information needed to estimate the number of steps required for correlation in the spatial Markov model to no longer be important. In this case it appears to be on the order of about 12 to 15 . Thus, if one measured the transition time over $15 \mathrm{~L}$ one could in principle build a predictive uncorrelated model using this transition time distribution and a jump size of $15 L$. Consistently, if one applies (16) to the travel time distributions obtained after a distance of $15 \mathrm{~L}$ one obtains a much better prediction of the asymptotic dispersion behaviors as shown by the corresponding results in figure 13 (magenta square symbols).

\section{Conclusions}

We have studied dispersion in a periodic wall bounded domain with sinusoidal boundaries using finite element numerical simulations of the flow field and random walk numerical simulations of transport. We have considered systems with Reynolds numbers that are sufficiently large that inertial effects play an important role on transport, but limited ourselves to systems where flows are laminar and steady. As Reynolds number increases in the chosen flow domain, recirculation zones emerge and become increasingly large in size as inertial effects grow. These recirculation zones act as traps for solute and cause a strong preferential channel down the center of the domain. In combination these effects strongly affect the nature and magnitude of dispersion in such a channel. To 

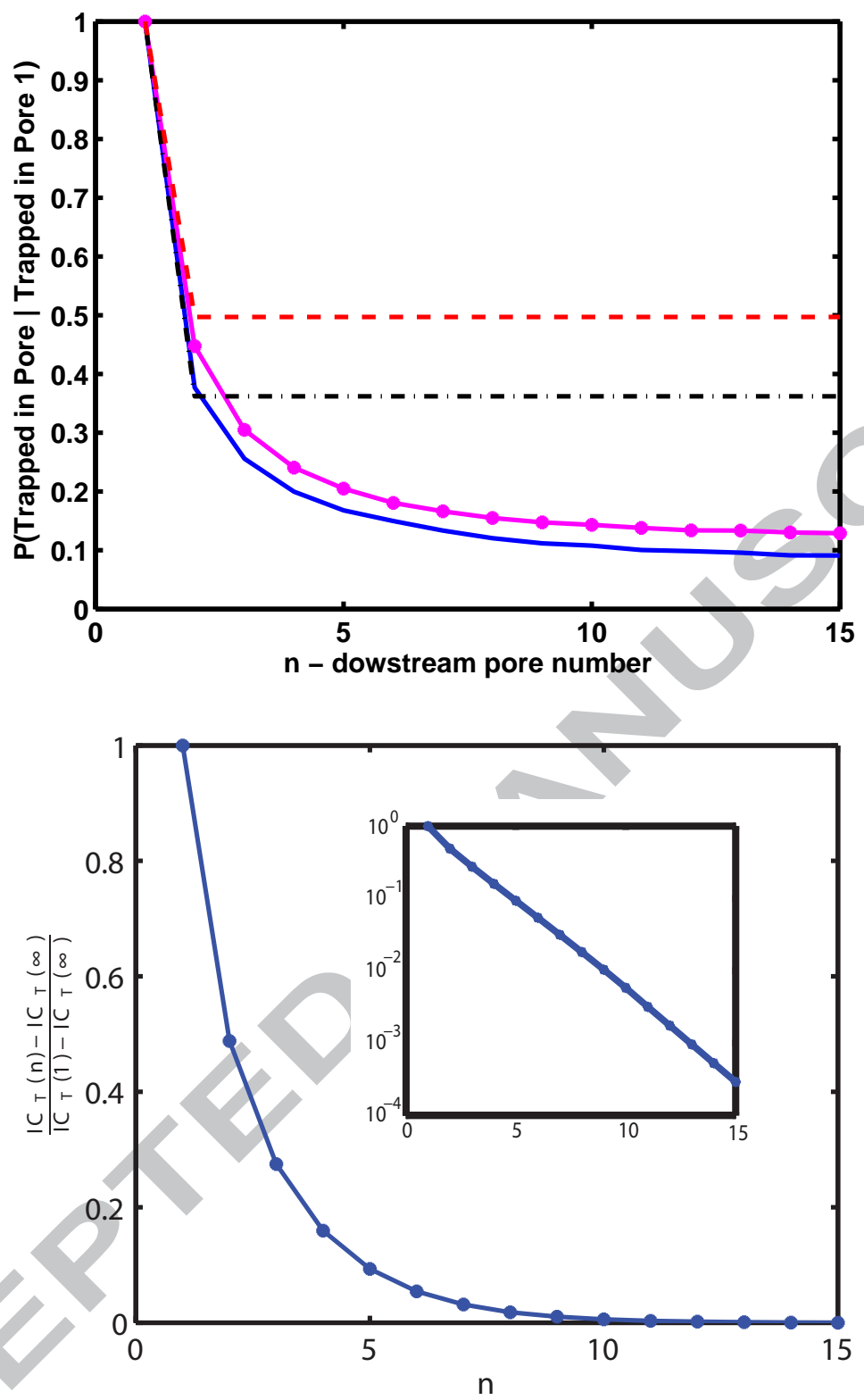

Figure 15: (Top) Probability of being trapped in downstream recirculation zone n, given that a particle is trapped in recirculation zone 1 . (Red-) $\mathrm{Re}=100, \mathrm{Pe}=100$, (Black -.) $\mathrm{Re}=30$, $\mathrm{Pe}=100,($ Purple -*) $\mathrm{Re}=100, \mathrm{Pe}=1000$ and (Blue - ) $\mathrm{Re}=30, \mathrm{Pe}=1000$. (Bottom) Normalized Importance of Correlation IC for successive powers of the transition matrix $T^{n}$ demonstrating the decorrelation over successive jumps. This is for $\mathrm{Re}=100$ and $\mathrm{Pe}=1000$. The inset shows the same data on a semi-log plot to highlight the exponential decay. 
date most research on such effects has been restricted to studying asymptotic dispersion effects, in analogy with Taylor dispersion.

In order to better understand and model preasymptotic dispersion, which cannot be effectively modeled using a classical Fickian approach, we develop a spatial Markov model, which aims to effectively model transport from early to asymptotic times. The method relies on measuring breakthrough times at two successive lengths and with this information calculating a correlation matrix that quantifies correlation between successive transit time jumps. In order to quantify the magnitude of this correlation we have defined a new parameter $I C$, the importance of correlation. $I C$, for this chosen configuration, suggests that correlation effects are unimportant when $P e<O(100)$, but become increasingly important as the Péclet number grows larger than this. Indeed we demonstrate that an effective model, which does not incorporate correlation between successive jumps, does an almost equally good job as a correlated equivalent of predicting breakthrough curves at several downstream breakthrough locations as well as in predicting the evolution of a plume's second centered moment over time when $P e=100$. However, when $P e=1000$ only the correlated model successfully matches observations, with the uncorrelated CTRW underpredicting the magnitude of the second centered moment and failing to accurately capture the early concentrations of the breakthrough curves. For a larger Péclet number of $P e=10^{5}$ this mismatch is even larger with the uncorrelated model failing to accurately capture both observed early and late times breakthrough curve arrivals, while the spatial Markov model successfully replicates observed behaviors. This demonstrates that for systems with smaller Péclet numbers, for this configuration less than 100, effective models need not include correlation effects and that conventional approaches may work well. Likewise though, if Péclet numbers are larger than this, correlation effects must be incorporated to reliably recover true behaviors. This reflects the fact that diffusion at the small scale is the process that decorrelates successive steps; thus larger relative diffussion effects mean less correlation.

To conclude the paper we explain the previously unexplained phenomenon 
where it has previously been observed that, for fixed Péclet number, the magnitude of the asymptotic dispersion coefficient grows rapidly with increasing advective effects, but then saturates at a more or less constant value beyond which little further increase occurs despite continued increases in Reynolds number. We do so using classical CTRW theory that does not incorporate correlations. Additionally we discuss how the transition matrix and the importance of correlation parameter can be used to estimate the probability of successive trapping events as the number of steps required such that correlation effects become unimportant.

While the presented geometry is incredibly simple it provides valuable insights into more complex systems, where both spatial Markov and uncorrelated CTRW models have been applied in the past. For these more complex systems it still most likely holds true that there is a critical Péclet number below which correlation effects may be neglected and above which they are critical in effective model development. Identifying such critical cutoffs will be important to improved future model development.

Acknowledgments DB would like to express thanks for financial support via Army Office of Research grant W911NF1310082 and NSF grant EAR-1113704. 


\section{References}

\section{References}

[1] De Anna, P. , T. Le Borgne, M. Dentz, A.M. Tartakovsky, D. Bolster and P. Davy, Flow Intermittency, Dispersion, and Correlated Continuous Time Random Walks in Porous Media Physical Review Letters (2014) 110184502

[2] Appuhamillage, T. A., V. A. Bokil, E. Thomann, E. Waymire, and B. D. Wood, Solute transport across an interface: A Fickian theory for skewness in breakthrough curves, Water Resources Research, (2010) 46, W07511, doi:10.1029/2009WR008258.

[3] R. Aris, On the dispersion of solute in a fluid flowing through a tube, Proceedings of the Royal Society of London. Series A, 235 (1956) 67-77.

[4] R. Aris, The longitudinal diffusion coefficient in flow through a tube with stagnant pockets, Chemical Engineering Science, (1959) 11, 194-198,

[5] S. Bachu, Screening and ranking of sedimentary basins for sequestration of $\mathrm{CO}_{2}$ in geological media in response to climate change., Environmental Geology 44 (2003) 277-289.

[6] D. Benson, R. Schumer, S. Wheatcraft and M. M Meerschaert. Fractional dispersion, Levy motion, and the MADE tracer tests, Transport in Porous Media 42 (2001) 211-240.

[7] D. Benson, S. Wheatcraft, M. M Meerschaert, The fractional-order governing equation of levy motion, Water Resources Research 36 (2000) 14131423.

[8] B. Berkowitz, A. Cortis, M. Dentz, H. Scher, Modeling non-Fickian transport in geological formations as a continuous time random walk, Reviews in Geophysics 44 (2006) RG2003. 
[9] B. Berkowitz, J. Zhou, Reactive solute transport in a single fracture, Water Resources Research 32 (4) (1996) 901-913.

[10] D. Bolster, T. L. Borgne, M. Dentz, Solute dispersion in channels with periodically varying apertures, Physics of Fluids 21 (2009) 056601.

[11] D. Bolster, M. Barahona, M. Dentz, D. Fernandez-Garcia, X. SanchezVila, P. Trinchero, C. Valhondo and D.M. Tartakovsky, Probabilistic risk analysis of groundwater remediation strategies, Water Resources Research, 45 (2006) W06413

[12] D. Bolster, F. Valdes-Parada, T. Le Borgne, M. Dentz, J. Carrera, Mixing in confined stratified aquifers, Journal of Contaminant Hydrology 120-121 (2011) 198-212.

[13] T. Le Borgne, M. Dentz, J. Carrera, Lagrangian statistical model for transport in highly heterogeneous velocity fields, Physical Review Letters 101 (2008) 090601.

[14] T. Le Borgne, M. Dentz, J. Carrera, Spatial Markov processes for modeling Lagrangian particle dynamics in heterogeneous porous media, Physical Review E 78 (2008) 026308.

[15] J. Bouquain, Y. Meheust, D. Bolster, P. Davy, The impact of inertial effects on solute dispersion in a channel with periodically varying aperture, Physics of Fluids 24 (2012) 083602.

[16] J. Bouquain, Y. Meheust, P. Davy, Horizontal pre-asymptotic solute transport in a model fracture with significant density contrasts, Journal of Contaminant Hydrology 120-121 (2011) 184-197.

[17] H. Brenner, Dispersion resulting from flow through spatially periodic porous media, Philosophical Transactions of the Royal Society of London. Series A 297 (1980) 81-133. 
[18] M. Cardenas, Direct simulation of pore level Fickian dispersion scale for transport through dense cubic packed spheres with vortices, Geochemistry, Geophysics, Geosystems 10 (2009) Q12014.

[19] M. Cardenas, D. Slottke, R. Ketcham, J. Sharp, Effects of inertia and directionality on flow and transport in a fracture, Journal of Geophysical Research-Solid Earth 114 (2009) B06204.

[20] J. Carrera, X. Sánchez-Vila, I. Benet, A. Medina, G. Galarza, J. Guimer, On matrix diffusion: formulations, solution methods, and qualitative effects, Hydrog. J. 6 (1998) 178-190.

[21] P. C. Chatwin. The approach to normality of concentration distribution of a solute in a solvent flowing along a straight pipe. Journal of Fluid Mechanics, (1970)43:321-352.

[22] K. Chaudhary, M. Cardenas, W. Deng, P. Bennett, Pore geometry effects on intrapore viscous to inertial flows and on effective hydraulic parameters, Water Resources Research 49 (2013) 1149-1162.

[23] J.M.P.Q. Delgado. Longitudinal and transverse dispersion in porous media. Chemical Engineering Research and Design, (2007), 85 (A9):1245-1252.

[24] M. Dentz, B. Berkowitz, H. Scher, Transport behavior of coupled continuous-time random walks, Physical Review E 78 (2008) 041110.

[25] M. Dentz, D. Bolster, Distribution- Versus Correlation-Induced Anomalous Transport in Quenched Random Velocity Fields, Physical Review Letters, (2010), 105,244301

[26] J.R. de Dreuzy, A. Beaudoin, J. Erthel, Asymptotic dispersion in 2D heterogeneous porous media determined by parallel numerical simulations, Water Resources Research, (2007), 43, W10439, doi:10.1029/2006WR005394.

[27] L. Donado, X. Sánchez-Vila, M. Dentz, J. Carrera, D. Bolster, Multicomponent reactive transport in multicontinuum media, Water Resources Research 45 (2009) W11402, doi:10.1029/2008WR006823. 
[28] B. Dykaar, P. Kitanidis, Macrotransport of a biologically reacting solute through porous media, Water Resources Research 32 (1996) 307-329.

[29] W. Gill, R. Sankarasubramanian, Exact analysis of unsteady convective diffusion, Proceeding of the Royal Society of London, Series A 316 (1970) 341.

[30] R. Haggerty, S. Gorelick, Multiple-rate mass transfer for modeling diffusion and surface reactions in media with pore scale heterogeneity, Water Resources Research 31 (1995) 2383-2400.

[31] U. Hornung, Homogenization and Porous Media, Springer, 1997.

[32] S.C. James, C. V. Chrysikopoulos, An efficient particle tracking equation with specified spatial step for the solution of the diffusion equation, Chemical Engineering Science, (2001) 56(23), 6535-6543

[33] P.K. Kang, M. Dentz, T. Le Borgne, R. Juanes, Spatial Markov model of anomalous transport through random lattice networks. Physical Review Letters, 107 (2011), 180602.

[34] K. Katayama, H. Nomura, H. Ogata and T. Eitoku. Diffusion coefficients for nanoparticles under flow and stop-flow conditions. Physical Chemistry Chemical Physics, 11 (2009) 10494-10499

[35] P. Kitanidis, B. Dykaar, Stokes flow in a slowly varying two-dimensional periodic pore, Transport in Porous Media 26 (1997) 89-98.

[36] M. Latini, A. Bernoff, Transient anomalous diffusion in Poiseuille flow, Journal of Fluid Mechanics 441 (2001) 339.

[37] T. Le Borgne, M. Dentz, D. Bolster, J. Carrera, J.R. de Dreuzy and P Davy (2010) Non-Fickian mixing: temporal evolution of the scalar dissipation rate in porous media Advances in Water Resources, 33, 1468-1475, doi:10.1016/j.advwatres.2010.08.006 
[38] T. Le Borgne, D. Bolster, M. Dentz, P. de Anna, A. Tartakovsky, Effective pore-scale dispersion upscaling with a correlated CTRW approach, Water Resources Research 47 (2012) W12538.

[39] M. M. Meerschaert, E. Nane, Y. Xiao, Correlated continuous time random walks, Statistics \& Probability Letters 79 (9) (2009) 1194-1202.

[40] H. Moffat, Viscous and resistive eddies near a sharp corner, Journal of Fluid Mechanics 18 (1963) 1-18.

[41] O. Plumb, S. Whitaker, Dispersion in heterogeneous porous media: 1. local volume averaging and large-scale averaging, Water Resources Research 24 (1988) 913-926.

[42] M. C. Richmond, W. A. Perkins, T. D. Scheibe, A. Lambert, B. D. Wood, Flow and axial dispersion in a sinusoidal-walled tube: Effects of inertial and unsteady flows, Advances in Water Resources, 62 (B), 215-226.

[43] M. Shlesinger, Asymptotic solutions of continuous-time random walks, Journal of Statsistical Physics 10 (1974) 421-433.

[44] G. I. Taylor, Dispersion of soluble matter in solvent flowing slowly through a tube, Proceedings of the Royal Society of London. Series A, 219 (1953) 186-203 186-203.

[45] V. Vilarrasa, D. Bolster, M. Dentz, S. Olivella, J. Carrera, Effects of $\mathrm{CO}_{2}$ compressibility on $\mathrm{CO}_{2}$ storage in deep saline aquifers, Transport in Porous Media 85 (2010) 619-639.

[46] B.D. Wood. Inertial effects on dispersion in porous media. Water Resources Research, 43, (2007), W12S16:doi:10.1029/2006WR005790.

[47] B.D. Wood and F. J. Valdes-Parada. Volume averaging: Local and nonlocal closures using a green's function approach. Advances in Water Resources, (2013) 51:139-167. 
[48] M. Willman, J. Carrera, X. Sánchez-Vila, O. Silva, M. Dentz, Coupling of mass transfer and reactive transport for non-linear reactions in heterogeneous media, Water Resources Research, 46, W07512, doi:10.1029/2009WR007739.

[49] J.S. Yu. Dispersion in laminar flow through tubes by simultaneous diffusion and convection. Journal of Applied Mechanics, (1981), 48:217-223. 
- We study preasymptotic transport in a simple pore geometry

- Inertial flow effects are important and lead to strong recirculation zones.

- We test a Spatial Markov model to predict observed transport

- Correlation effects are important when Peclet number is larger than $O(100)$. 